\title{
Thickness-Dependent Secondary Structure Formation of Tubelike Polymers
}

Thomas Vogel ${ }^{1}$ (a), Thomas Neuhaus ${ }^{2}$ (b), Michael Bachmann ${ }^{1}$ (c) and Wolfhard Janke ${ }^{1}$ (d)

Institut für Theoretische Physik and Centre for Theoretical Sciences (NTZ), Universität Leipzig, Postfach 100 920,

D-04009 Leipzig, Germany

2 John von Neumann Institute for Computing (NIC), Forschungszentrum Jülich, D-52425 Jülich, Germany

PACS 05.10.-a - Computational methods in statistical physics and nonlinear dynamics

PACS 87.15.Aa - Theory, modeling, and computer simulation

PACS 87.15.Cc - Folding: thermodynamics, statistical mechanics, models, and pathways

\begin{abstract}
By means of sophisticated Monte Carlo methods, we investigate the conformational phase diagram of a simple model for flexible polymers with explicit thickness. The thickness constraint, which is introduced geometrically via the global radius of curvature of a polymer conformation, accounts for the excluded volume of the polymer and induces cooperative effects supporting the formation of secondary structures. In our detailed analysis of the temperature and thickness dependence of the conformational behavior for classes of short tubelike polymers, we find that known secondary-structure segments like helices and turns, but also ringlike conformations and stiff rods are dominant intrinsic topologies governing the phase behavior of such cooperative tubelike objects. This shows that the thickness constraint is indeed a fundamental physical parameter that allows for a classification of generic polymer structures.
\end{abstract}

Introduction. - Resolving structural properties of single molecules is a fundamental issue as molecular functionality strongly depends on the capability of the molecules to form stable conformations. Experimentally, the identification of substructures is typically performed, for example, by means of single-molecule microscopy, Xray analyses of polymer crystals, or NMR for polymers in solution. With these methods, structural details of specific molecules are identified, but these can frequently not be generalized systematically with respect to characteristic features being equally relevant for different polymers.

Therefore, the identification of generic conformational properties of polymer classes is highly desirable. The todate most promising approach to attack this problem is to analyze polymer conformations by means of comparative computer simulations of polymer models on mesoscopic scales, i.e., by introducing relevant cooperative degrees of freedom and additional constraints. In a typical modeling approach of this kind, the linear polymer is considered

\footnotetext{
(a) E-mail: Thomas.Vogel@itp.uni-leipzig.de

(b) E-mail: neuhaus@physik.uni-bielefeld.de

(c) E-mail: Michael.Bachmann@itp.uni-leipzig.de

(d) E-mail: Wolfhard.Janke@itp.uni-leipzig.de, Homepage: www.physik.uni-leipzig.de/CQT.html
}

as a chain of beads and springs, where the monomeric properties, e.g., caused by the side chain, are accumulated in an effective, specifically parametrized single interaction point of dimension zero ("united atom approach"). The linear extension of the chain is accounted for by introducing springs or stiff bonds to mimic covalent bonds in an effective way. Noncovalent van der Waals interactions between pairs of monomers are typically modeled by Lennard-Jones (LJ) potentials. In such models, only the repulsive short-range part of the LJ potentials keeps pairs of monomers apart. Such models have proven to be quite useful in identifying universal aspects of global structure formation processes. Examples include the characterization of folding channels known from natural proteins [1] and coupled binding-folding aggregation phenomena [2], but are by no means limited to this specific sort of polymers.

For the identification of underlying secondary structure segments like helices, strands, and turns as ground states, however, the modeling of volume exclusion by means of pure LJ pair potentials is not sufficient to form clearly distinct secondary structures enabling a classification scheme. Segments of such secondary structures were found, e.g., in dynamical LJ polymer studies of transient 
states occurring in the collapse process [3] or as ground states in models with stiffness [4], explicit hydrogen bonding $[5,6]$, or explicit solvent particles $[7,8]$. It could also be shown that helical structures form by introducing anisotropic monomer-monomer potentials in conjunction with a wormlike backbone model [9] or by combining excluded volume and torsional interactions [10].

The formation of secondary structures requires cooperative behavior of adjacent monomers, i.e., in addition to pairwise repulsion, information about the relative position of the monomers to each other in the chain is necessary to effectively model the competition between noncovalent monomeric attraction and short-range repulsion due to volume exclusion effects [11]. The simplest way to achieve this in a general, mesoscopic model is to introduce a hard single-parameter thickness constraint and, thus, to consider a polymer chain rather as a three-dimensional tubelike object than as a one-dimensional, linelike string of monomers $[12,13]$. Note that this approach differs significantly from frequently studied cylindrical tube models [14], where the tube thickness only mimics volume exclusion but not cooperativity such that explicit modeling of hydrogen bonds is required to generate secondary structures.

Model and Methods. - In this Letter, we analyze the general thermodynamic (pseudo)phase diagram of secondary polymer structures in dependence of the thickness constraint. The thickness will, therefore, be considered as coupling parameter that separates the different conformational phases polymers generally can reside in. A natural choice for parametrizing the thickness of a polymer conformation with $N$ monomers, $\mathbf{X}=\left(\mathbf{x}_{1}, \ldots, \mathbf{x}_{N}\right)$, is the global radius of curvature $r_{\mathrm{gc}}$ [15]. It is defined as the radius $r_{\mathrm{c}}$ of the smallest circle connecting any three different monomer positions $\mathbf{x}_{i}, \mathbf{x}_{j}, \mathbf{x}_{k}(i, j, k=1, \ldots, N)$ :

$$
r_{\mathrm{gc}}(\mathbf{X})=\min \left\{r_{\mathrm{c}}\left(\mathbf{x}_{i}, \mathbf{x}_{j}, \mathbf{x}_{k}\right) \forall i, j, k \mid i \neq j \neq k\right\} .
$$

Denoting the distance between two points by $r_{i j}=\left|\mathbf{x}_{i}-\mathbf{x}_{j}\right|$ and the area of the triangle spanned by any three points by $A_{\Delta}\left(\mathbf{x}_{i}, \mathbf{x}_{j}, \mathbf{x}_{k}\right), r_{\mathrm{c}}$ is given as

$$
r_{\mathrm{c}}=\frac{r_{i j} r_{j k} r_{i k}}{4 A_{\Delta}\left(\mathbf{x}_{i}, \mathbf{x}_{j}, \mathbf{x}_{k}\right)} .
$$

With these definitions, the polymer tube $\mathbf{X}$ has the "thickness" (or diameter) $d(\mathbf{X})=2 r_{\mathrm{gc}}(\mathbf{X})$ which is illustrated in an intuitive way in Refs. $[15,16]$.

We here consider linear, flexible polymers with stiff bonds of unit length $\left(r_{i+1}=1\right)$. The pairwise interactions among nonbonded monomers are modeled by a standard LJ potential and thus the energy of a conformation $\mathbf{X}$ reads

$$
E(\mathbf{X})=\sum_{i, j>i+1} V_{\mathrm{LJ}}\left(r_{i j}\right),
$$

where $V_{\mathrm{LJ}}\left(r_{i j}\right)=4 \varepsilon\left[\left(\sigma / r_{i j}\right)^{12}-\left(\sigma / r_{i j}\right)^{6}\right]$. By setting $\sigma=$ $1, V_{\mathrm{LJ}}\left(r_{i j}\right)$ vanishes for $r_{i j}=1$ and is minimal at $r_{i j}^{\min }=$ $2^{1 / 6} \approx 1.122$.
Since we are interested in classifying conformational pseudophases of polymers with respect to their thickness, we introduce the restricted conformational space $\mathcal{R}_{\rho}=\left\{\mathbf{X} \mid r_{\mathrm{gc}}(\mathbf{X})>\rho\right\}$ of all conformations $\mathbf{X}$ with a global radius of curvature larger than a thickness constraint $\rho$, which can be understood as an effective measure for the extension of the polymer side chain. Given $\rho$, obviously only conformations with $r_{\mathrm{gc}} \geq \rho$ can occur.

The canonical partition function of the restricted conformational space thus reads

$$
Z_{\rho}=\int \mathcal{D} X \Theta\left(r_{\mathrm{gc}}(\mathbf{X})-\rho\right) e^{-E(\mathbf{X}) / k_{\mathrm{B}} T},
$$

where $k_{\mathrm{B}} T$ is the thermal energy (we use units in which $\varepsilon=k_{\mathrm{B}}=1$ in the following) and $\Theta(z)$ is the Heaviside function. In this thickness-restricted space, canonical statistical averages of any quantity $O$ are then calculated via $\langle O\rangle_{\rho}=Z_{\rho}^{-1} \int \mathcal{D} X O(\mathbf{X}) \Theta\left(r_{\mathrm{gc}}(\mathbf{X})-\rho\right) \exp \left[-E(\mathbf{X}) / k_{\mathrm{B}} T\right]$.

To characterize the phase diagram, we have first performed exhaustive energy-landscape paving (ELP) optimizations [17] in order to identify lowest-energy conformations as reference states of flexible polymers under the constraint of a given minimal global radius of curvature $\rho$. Next, we have analyzed the interplay of structural and thermal properties of polymers with $N=8, \ldots, 13$ monomers for a very large number of $\rho$ values. It should be emphasized that our detailed thermodynamic analysis aiming at the entire structural phase diagram requires precise datasets that can only be obtained by means of sophisticated generalized-ensemble methods. We have employed parallel tempering [18], multicanonical sampling [19], and the Wang-Landau method [20], and compared the results. In the following, we shall focus on the tube polymer with $N=9$ monomers as most of the observed features of this 9 mer are generic and thus also common to the longer chains.

Results and Discussion. - Figure 1 shows the ground-state energy per monomer as a function of $\rho$ (with bin sizes $\Delta \rho \leq 0.01$ in the most interesting region). Also shown are lowest-energy conformations for exemplified values of $\rho$. The ground-state energy per monomer for the linelike 9 mer (i.e., $\rho=0$ ) is in our units $E_{\min } / N=-1.85$. The thickness constraint becomes relevant, if $\rho$ is larger than half the characteristic length scale $r_{i j}^{\min }$ of the LJ potential: In the interval $2^{-5 / 6} \approx 0.561<\rho<\rho_{\alpha} \approx 0.686$ conformations are pre-helical. The nonbonded interaction distance is still allowed to be so small that structures are deformed. Nonetheless, the onset of helix formation is clearly visible as it is an intrinsic geometrical property of any linelike object. Optimal space-filling helical symmetry is reached when approaching $\rho_{\alpha}$, where the ground-state conformation takes the perfect $\alpha$-helical shape (see inset of Fig. 11). All torsional angles are identical (near $41.6^{\circ}$ ) and also all local radii are constant; the number of monomers per winding is 3.6. Note that for proteins, where the effective distance between two $\mathrm{C}^{\alpha}$ atoms is about $3.8 \AA, \rho_{\alpha}$ 

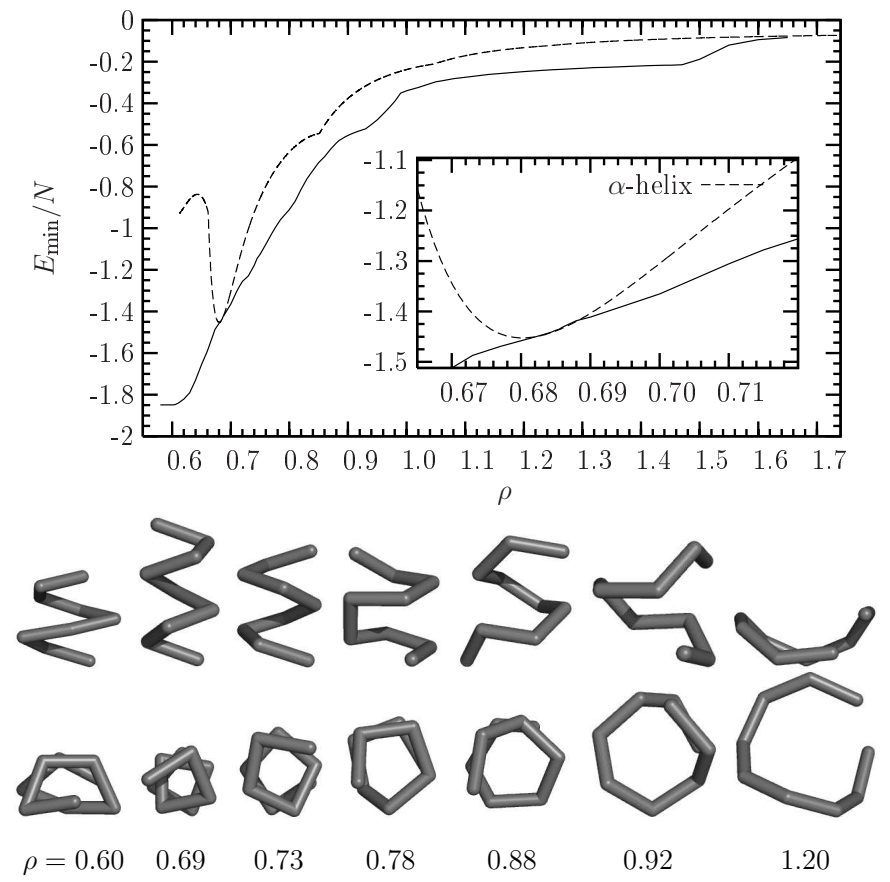

Fig. 1: Ground-state energy per monomer $E_{\min } / N$ of tubelike polymers with nine monomers as a function of the global radius of curvature constraint $\rho$ (solid line). For comparison, also the energy curve of the perfect $\alpha$-helix is plotted (dashed line). The inset shows that for a small interval around $\rho \approx 0.686$, the ground-state structure is perfectly $\alpha$-helical. Also depicted are side and top views of putative ground-state conformations for various exemplified values of $\rho$. For the purpose of clarity, the conformations are not shown with their natural thickness.

in our units corresponds indeed to a pitch of about $5.4 \AA$ as known from $\alpha$-helices of proteins. Thus, an $\alpha$-helix is a natural geometric shape for tubelike polymers. Hydrogen bonds stabilize these structures in nature - but are not a necessary prerequisite for forming such secondary structures.

For larger values of $\rho$, helices unwind, i.e., the pitch gets larger and the number of monomers per winding increases. However, helical structures still dominate the ground-state conformations. It should be noted that our model is energetically invariant under helicity reversal, i.e., left-handed helices or segments are not explicitly disfavored and are, therefore, also equally present in the conformational space. In the interval $\rho_{\alpha} \leq \rho \lesssim 0.92$, fluctuation peaks of the derivative $\mathrm{d} E_{\min } / \mathrm{d} \rho$ (not shown) indicate that there are also stable helical conformations in the vicinity of $\rho \approx 0.73$ (winding number $\approx 4.5$ ) and $\rho \approx 0.78$ (winding number $\approx 5.0$ ). Near $\rho \approx 0.92$, the final helical state has been reached. The thickness has increased in such a way that the most compact conformation is a helix with a single winding. After that, a topological change occurs and the ground-state conformations are getting flatter. The helix finally opens up and planar conformations with similarities to $\beta$-hairpins become dominant. These structures are still stabilized by nonbonded LJ interactions between pairs of monomers. Increasing the thickness further leads to a breaking of these contacts and ringlike conformations become relevant [16]. We have verified that for values $r_{\mathrm{gc}} \approx N / 2 \pi$, ground-state conformations are almost perfect circles with radius $r_{\mathrm{gc}}$. The existence of ringlike conformations is a consequence of the long-range monomermonomer attraction. Eventually, for $\rho \rightarrow \infty$, the effective stiffness increases, also the end contacts disappear, and only thick rods are still present.

After these preparatory considerations of ground-state properties, we are now going to discuss the thermodynamic behavior of the tube polymers. Based on the peak structure of the specific heat as a function of temperature $T$ and thickness constraint $\rho$, we identify the structure of the conformational $\rho-T$ pseudophase diagram. We do this again for the 9 mer which allows for a very precise analysis. Only for such a small system, hundreds of separate generalized-ensemble computer simulations can be performed. However, we verified the results also for larger polymers with up to 13 monomers and found that there are no significant changes in the phase-diagram topology [21]. Even the expected shifts of the transition lines due to finite-length corrections are very small such that we have good reason to assume that the pseudophase diagram of the 9mer reflects the general phase structure of short tubelike polymers pretty well. This is partly due to the fact that the polymer thickness as defined via the global radius of curvature is a length-independent constraint and the chains in our study are short enough to prevent the formation of tertiary structures (as, e.g., arrangements of different secondary-structure segments forming a tertiary domain). For longer chains, however, tertiary structures are definitely relevant. The longest chain in our study, the polymer with $N=13$ monomers, already exhibits first indications of structure formation on globular length scales. However, the description of such tertiary folding processes is not in the focus of the present work. Compact globular conformations form by decreasing the temperature below the $\Theta$ point whose properties for tubelike polymers are a study worth in its own right.

Our main results are contained in the phase diagram of Fig. 2. which shows the specific-heat landscape $C_{V}(\rho, T)=$ $\left(\left\langle E^{2}\right\rangle_{\rho}-\langle E\rangle_{\rho}^{2}\right) / T^{2}$ for a 9mer as obtained from reweighting the density of states for given thickness constraint $\rho$. Dark regions correspond to strong energetic fluctuations, i.e., the darker the region the larger is the specific-heat value. Data points $(+)$ mark the peaks or ridges of the profile and indicate conformational activity and thus represent transitions between different conformational pseudophases. Error bars are not shown for clarity but are sufficiently small (for most data points smaller than symbol size), so that the identified pseudophase boundaries are statistically significant.

Guided by the analyses of the ground-state properties, 

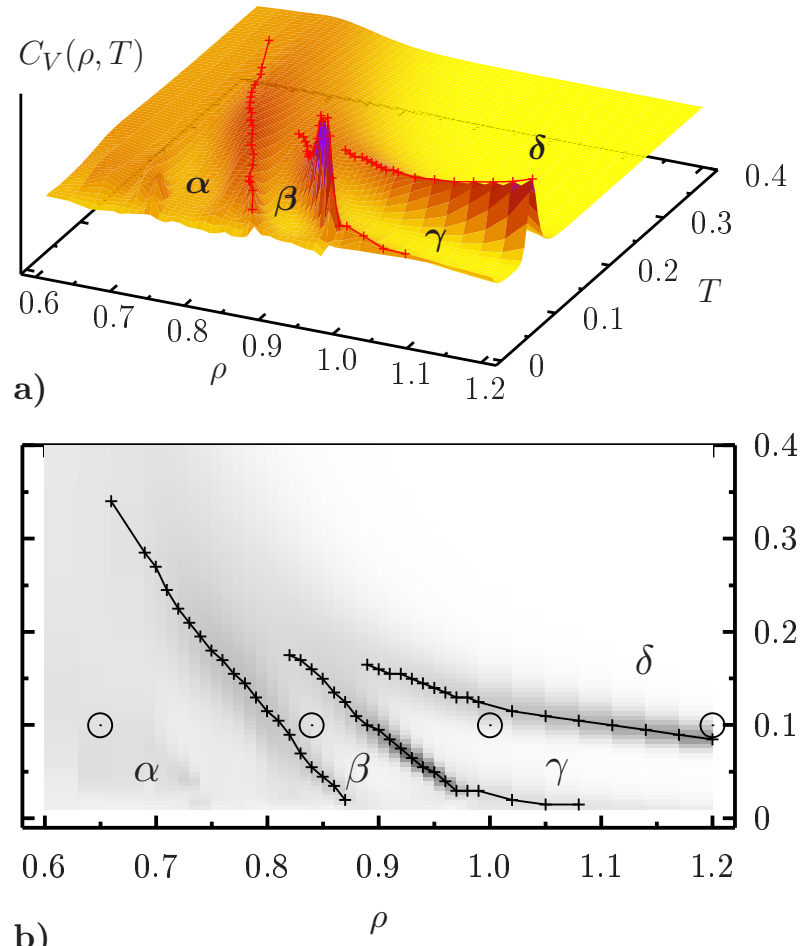

b)

Fig. 2: (a) Perspective and (b) projected view of the specificheat profile $C_{V}(\rho, T)$ for a 9 mer which is interpreted as structural pseudophase diagram of thermodynamically relevant tube polymer conformations in thickness-temperature parameter space. Dark regions and data points $(+)$ indicate the ridges of the landscape and separate conformational phases.

Helical or helix-like conformations dominate in region $\alpha$, sheets in region $\beta$, rings in region $\gamma$, and stiff rods in pseudophase $\delta$. Circles $(\odot)$ indicate the locations where the exemplified conformations of Table 1 are relevant. The general structure of the phase diagram remains unchanged also for the longer polymers considered in our study.

we identify four principal pseudophases 1 . In region $\alpha$, helical conformations are the most relevant structures. In particular, the $\alpha$-helix resides in this pseudophase. Characteristic for the transition from pseudophase $\alpha$ to $\beta$ is the unwinding of the helical structures which are getting more planar. Thus, region $\beta$ is dominated by simple sheetlike structures. Since the 9 mer is rather short, the only sheet-like class of conformations is the hairpin. For longer chains, one also finds more complex sheets, e.g., lamellar structures $[12,21]$. A characteristic property of the hairpins is that these are still stabilized by nonbonded interactions. These break with larger thickness and higher temperature. Entering pseudophase $\gamma$, dominating structures possess ringlike shapes. Finally, region $\delta$ is the phase of random coils, which are getting stiffer for large thickness

\footnotetext{
${ }^{1}$ We note that there are singular points in the parameter space corresponding to special geometric representations of secondary structures. For the chain with length $N=8$ and $r_{\mathrm{gc}} \approx 1 / \sqrt{2}$, for example, the degenerate ground-state conformation exhibits an almost perfect alignment of the chain along the edges of a cube.
}

Table 1: Exemplified conformations being thermodynamically relevant in the respective pseudophases shown in Fig. 2, visualized in different representations.

phase type

and eventually resembling rods. Representative polymer conformations dominating the pseudophases in the regions $\alpha$ to $\delta$ are depicted in Table 1 in different representations.

Summary. - In this Letter we have focused on an analysis of the thermodynamic properties of tubelike polymers. The tube picture is a simplification of the volume extension of polymers due to steric constraints of their backbone or the presence of side chains. The thickness of such a mesoscopic tube can be considered as a single steric parameter that induces cooperative effects and permits the discrimination of polymers. Thus, the phase diagram presented here does not only allow for the classification of possible thermodynamic conformational phases of a single polymer with fixed thickness. Rather, performing generalized-ensemble simulations for different thicknesses enabled us for the first time to resolve the complete (pseudo)phase behavior with respect to the thickness constraint and temperature. This means that the we have identified the generic structure of the conformational phase space at non-zero temperatures for classes of polymers, parametrized by their thickness. Although we employ a mesoscopic model for flexible polymers, we find that the thickness constraint is an intrinsic source of an effective stiffness and enhances the capability of a polymer to form secondary structures which are stable against thermal fluctuations. The stability limits for increasing temperature are elucidated in the phase diagram in Fig. 2 which summarizes our main findings. In particular, we clearly find helical and sheet-like structures which are dominant in different pseudophases. Thus, the thickness is indeed a fun- 
damental physical parameter that allows for the classification of polymers with respect to their transition behavior and their preference to form characteristic secondary structures, depending on external parameters such as temperature.

Since the development of high-resolution experimental techniques is breathtakingly advancing, the interest in structural properties on nanoscopic scales is increasing, in particular when it comes to applications where small molecules are used as building blocks in the design of functional molecular machines. In these cases the understanding of the effect of sterically induced constraints on molecular structure formation is of particular importance.

This work is partially supported by the DFG (German Science Foundation) under Grant Nos. JA 483/24-1/2 and the Graduate School of Excellence "BuildMoNa". Some simulations were performed on the supercomputer JUMP of the John von Neumann Institute for Computing (NIC), Forschungszentrum Jülich, under Grant No. hlz11.

\section{REFERENCES}

[1] Schnabel S., Bachmann M. and Janke W., Phys. Rev. Lett., 98 (2007) 048103; J. Chem. Phys., 126 (2007) 105102.

[2] Junghans C., Bachmann M. and Janke W., Phys. Rev. Lett., 97 (2006) 218103; J. Chem. Phys., 128 (2008) 085103.

[3] Sabeur S. A., Hamdache F. and Schmid F., Phys. Rev. E, 77 (2008) 020802(R).

[4] Noguchi H. and Yoshikawa K., J. Chem. Phys., 109 (1998) 5070.

[5] Hoang T. X., Trovato A., Seno F., Banavar J. R. and Maritan A., Proc. Natl. Acad. Sci. USA, 101 (2004) 7960 .

[6] Wolff K., Vendruscolo M., and Porto M., Gene, 422 (2008) 47.

[7] Snir Y. and Kamien R. D., Science, 307 (2005) 1067; Phys. Rev. E, 75 (2007) 051114.

[8] Hansen-Goos H., Roth R., Mecke K. and Dietrich S., Phys. Rev. Lett., 99 (2007) 128101.

[9] Kemp J. P. and Chen Z. Y., Biomacromolecules, 2 (2001) 389.

[10] Rapaport D. C., Phys. Rev. E, 66 (2002) 011906.

[11] Banavar J. R., Flammini A., Marenduzzo D., Maritan A. and Trovato A., J. Phys.: Condens. Matter, 15 (2003) S1787.

[12] Banavar J. R. and Maritan A., Rev. Mod. Phys., 75 (2003) 23.

[13] Maritan A., Micheletti C., Trovato A. and BaNAVAR J. R., Nature, 406 (2000) 287.

[14] Auer S., Miller M. A., Krivov S. V., Dobson C. M., Karplus M. and Vendruscolo M., Phys. Rev. Lett., 99 (2007) 178104.

[15] Gonzalez O. and Maddocks J. H., Proc. Natl. Acad. Sci., 96 (1999) 4769.
[16] Neuhaus T., Zimmermann O. and Hansmann U. H. E., Phys. Rev. E, 75 (2007) 051803.

[17] Hansmann U. H. E. and Wille L. T., Phys. Rev. Lett., 88 (2002) 068105.

[18] Hukushima K. and Nemoto K., J. Phys. Soc. Jpn., 65 (1996) 1604; Geyer C. J., Computing Science and Statistics. Proceedings of the 23rd Symposium on the Interface., edited by Keramidas E. M. (Interface Foundation, Fairfax Station) 1991, p. 156.

[19] Berg B. A. and Neuhaus T., Phys. Lett. B, 267 (1991) 249; Phys. Rev. Lett., 68 (1992) 9.

[20] Wang F. and Landau D. P., Phys. Rev. Lett., 86 (2001) 2050 .

[21] Vogel T., Neuhaus T., Bachmann M. and Janke W., unpublished. 